Enhancement of infrared absorption of low-temperature uracil thin films by a nanostructured silver surface

A. Yu. Ivanov and S. G. StepanianL. AdamowiczV. A. Karachevtsev

Citation: Low Temperature Physics 42, 106 (2016); doi: 10.1063/1.4941965

View online: http://dx.doi.org/10.1063/1.4941965

View Table of Contents: http://aip.scitation.org/toc/ltp/42/2

Published by the American Institute of Physics

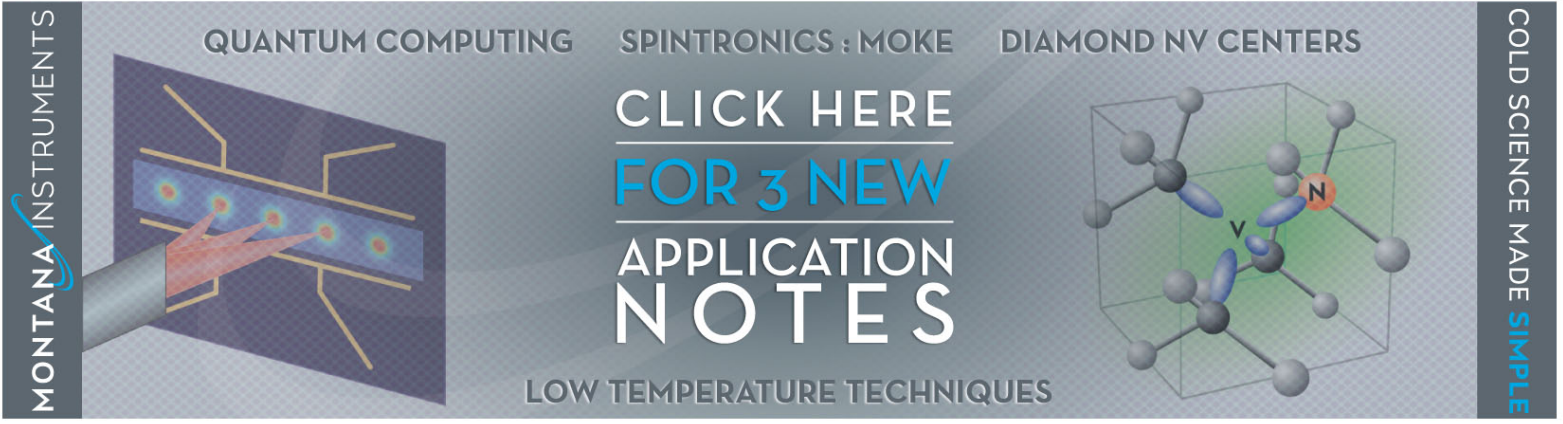




\title{
Enhancement of infrared absorption of low-temperature uracil thin films by a nanostructured silver surface
}

\author{
A. Yu. Ivanov ${ }^{\text {a) }}$ and S. G. Stepanian \\ B. Verkin Institute for Low Temperature Physics and Engineering of the National Academy of Sciences \\ of Ukraine, 47 Nauki Ave., Kharkov 61103, Ukraine \\ L. Adamowicz \\ Department of Chemistry and Biochemistry, University of Arizona, Tucson, Arizona 85721, USA
}

\section{A. Karachevtsev}

B. Verkin Institute for Low Temperature Physics and Engineering of the National Academy of Sciences of Ukraine, 47 Nauki Ave., Kharkov 61103, Ukraine

(Submitted November 12, 2015)

Fiz. Nizk. Temp. 42, 142-148 (February 2016)

\begin{abstract}
Enhancement of infrared absorption (SEIRA) of adsorbed biological molecules by a nanostructured metal surface is one of the main routes to increasing the sensitivity of modern optical biosensors. The FTIR absorption spectra of thin films of the RNA base uracil deposited on low-temperature substrate $(T=6 \mathrm{~K})$ with nanoscale silver structures were investigated in the spectral range $2700-600 \mathrm{~cm}^{-1}$. It was shown that the intensity of the absorption bands corresponding to $\nu \mathrm{CO}$ stretching vibrations (range $1800-1600 \mathrm{~cm}^{-1}$ ) of uracil (Ur) thin films increases 3-4 fold. For multilayer films, the influence of the nanostructures on the vibrational spectra weakens, and for the film layers more than $15 \mathrm{~nm}$ away from the surface, the enhancement is essentially absent. The energies and the vibrational spectra of the complexes of uracil monomers and dimers with 20-atom tetrahedral silver nanoclusters were calculated by the quantum-mechanical method DFT/B3LYP. The most stable complexes have the coordination bond between the top of the silver tetrahedron and the oxygen of the carbonyl group $\mathrm{C} 4 \mathrm{O}$. It was found that the formation of such complexes significantly (3-5 fold) enhances the intensity of the $\nu \mathrm{C} 4 \mathrm{O}$ stretching vibration of uracil, while the intensities of the $\beta \mathrm{NH}, \beta \mathrm{CH}$ and ring bending vibrations do not increase significantly. (C) 2016
\end{abstract}

AIP Publishing LLC. [http://dx.doi.org/10.1063/1.4941965]

\section{Introduction}

Development of ultra-sensitive biosensors based on the interaction between artificial nanostructures and biological molecules is one of the priority directions of the development of modern nanobiotechnology. Significant efforts are directed towards studying the phenomena of surface enhanced Raman scattering (SERS) and surface enhanced infrared absorption (SEIRA). ${ }^{1-4}$

Both phenomena could be interpreted in terms of electromagnetic and molecular enhancement mechanisms. The electromagnetic enhancement mechanism originates from local enhancement of the electromagnetic field near the structural inhomogeneities of a metal due to the interaction with the surface plasmons. Molecular or chemical enhancement mechanism is associated with a change in the dipole moments of specific vibrational transitions upon formation of the coordination bonds in the first layer of adsorbed molecules. Similar to SERS, ${ }^{5-7}$ the SEIRA phenomenon was first found on surfaces with stochastic structural inhomogeneities. ${ }^{8}$ On different surfaces the intensity enhancement of spectral bands by SEIRA ranges from 20 to 500 fold. ${ }^{8,9}$ Development of the electron-beam lithography techniques has made it possible to obtain the enhancing surfaces with nanostructures of a given size. ${ }^{10}$ The spectral and enhancing properties of surfaces with the nanostructures forming nanoantenna oscillators have been reported in Ref. 11 Experiments have shown that a nanoantenna or ordered arrays of nanoantennas can achieve a gain of $10^{4}-10^{5}$ for the IR spectral bands with the frequencies close to that of the plasmon resonance of the nanoantenna. ${ }^{11-14}$ Such enhancement provides sensitivity in the attomole range $\left(10^{-18} \mathrm{~mol}\right)$ in the IR region of the spectrum. ${ }^{11}$ However, the formation of a nanoantenna array is only possible by modern electron-beam lithography and the thermal and mechanical properties of the special substrates may hinder their use at low temperatures. In the literature there are no data on the study of biomolecules at low temperatures by SEIRA.

The aim of this research was to evaluate the enhancement ability of a low-temperature copper mirror with a nanostructured silver coating and the prospects for its use in the study of molecules isolated in low-temperature matrices of submicron thicknesses. To observe the SEIRA effect at temperatures below $10 \mathrm{~K}$, the absorption bands of the infrared spectra of uracil (Ur) have been selected. The uracil molecules have a low evaporation temperature and intense absorption bands of stretching vibrations of CO groups. ${ }^{16,17}$

\section{Experimental and computational methods}

Low-temperature experiment was carried out by using a matrix-isolation FTIR spectroscopy setup described previously. ${ }^{17-20}$ The FTIR spectra of uracil were obtained in the range of $2700-600 \mathrm{~cm}^{-1}$ with an apodized resolution of 
$2.5 \mathrm{~cm}^{-1}$. In the analysis of the spectra, the experimental data on the FTIR spectra of isolated Ur molecules obtained earlier for Ar matrices were used. ${ }^{17} \mathrm{~A}$ low-temperature quartz microbalance ${ }^{20}$ allowed to measure the molecular flow of uracil (Sigma Aldrich, USA) in the range of 10-20 $\mathrm{ng} /\left(\mathrm{s} \cdot \mathrm{cm}^{2}\right)$. To remove adsorbed water, the Ur powder was annealed in vacuum at a temperature of about $350{ }^{\circ} \mathrm{C}$. Evaporation of uracil from a Knudsen cell was carried out at a temperature of $390 \mathrm{~K}$. In contrast to the known procedures, ${ }^{15}$ silver was deposited electrochemically ${ }^{21}$ not on a gold layer, but on a mechanically polished copper mirror with a diameter of $2 \mathrm{~cm}$. Micrographs of the mirror surfaces were obtained with a scanning electron microscope JSM-820 (JEOL, Japan). During an experiment, uracil molecules were sequentially deposited on a polished copper mirror and a copper mirror with silver coating. The mirrors and microbalance were in placed in vacuum of $3 \times 10^{-7}$ Torr at a temperature of $6 \mathrm{~K}$. We prepared thin films of Ur $(10-50 \mathrm{~nm})$ to examine the SEIRA effect along with a $200 \mathrm{~nm}$-thick film to compare with the absorption spectra of Ur molecules isolated in Ar matrices.

For quantum chemical calculations the program Gaussian 09 was used. ${ }^{22}$

Optimization of molecular structures and vibrational spectra calculation were performed by the methods of density functional theory DFT/B3LYP with the basis set BS1: the extended basis 6-311++G(df,pd) for uracil and the basis with the effective core potentials Stuttgart RSC 1997 ECP for silver clusters. BSSE (Basis set superposition error) procedure for correction of the energy of a complex, which is standard for complexes, was not performed since the coordination bond of a length $2.4 \AA$ was formed between the components of the complex. The experimental and calculated absorption spectra were compared using SYNSPEC. ${ }^{23}$

\section{Results and discussion}

For studying the effect of the mirror surface on the spectrum, the region of the most intense $\mathrm{Ur}$ vibrations $1800-1200 \mathrm{~cm}^{-1}$ was selected (Fig. 1). Comparison of the absorption spectra obtained for Ur molecules isolated in $\mathrm{Ar}$ matrix and the spectra of the low-temperature amorphous film of this compound allowed us to extract the $\nu \mathrm{CO}$ stretching vibrations, as well as the stretching vibrations of the ring ( $\nu$ ring) and the $\beta \mathrm{NH}$ and $\beta \mathrm{CH}$ bending vibrations (Fig. 1). Using a quartz microbalance, the specific density of Ur samples was determined as $34 \times 10^{-6} \mathrm{~g} / \mathrm{cm}^{2}$, which corresponds to $18.3 \times 10^{16}$ molecules $/ \mathrm{cm}^{2}$. At this density of molecules, the Ur films consisted of about 500-700 layers. For the distance between the flat rings of Ur molecules of $3.4 \AA{ }^{24}$ this corresponds to a film thickness of $170-230 \mathrm{~nm}$. It should be noted that these estimates of the film thickness are rather approximate since they are made on the assumption that the preferred orientation of the plane of the pyrimidine ring of an Ur molecule is parallel to the surface. For warm Ur films, this orientation of the molecules was observed experimentally. ${ }^{25}$ However, deposition of the molecules on a mirror at $6 \mathrm{~K}$ leads to an amorphous structure of the film with disordered arrangement of the molecules and disordered structure of the intermolecular hydrogen bonds. Due to the amorphous nature of the film, the absorption bands are broadened. As a

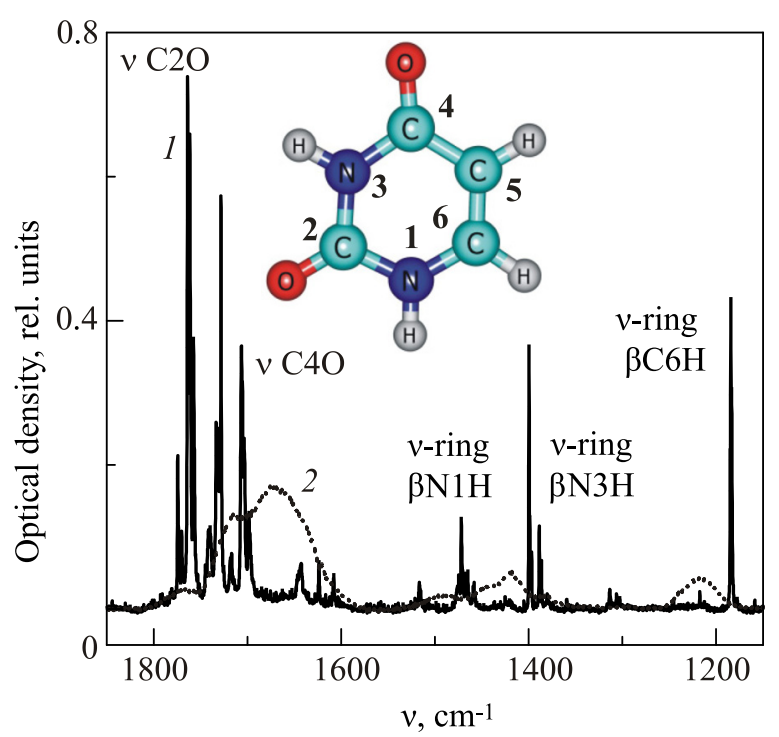

FIG. 1. Structure of an uracil molecule and FTIR absorption spectra in the region of the $\nu \mathrm{CO}$ stretching vibrations, stretching vibrations of the ring and bending vibrations: 1 - Ur molecules isolated in Ar matrix $(T=10 \mathrm{~K}$, concentration of 1:1000); $2-\mathrm{Ur}$ amorphous film at $T=6 \mathrm{~K}$. The spectra are renormalized to the equal concentration of Ur molecules of about $34 \times 10^{-6}$ $\mathrm{g} / \mathrm{cm}^{2}( \pm 10 \%)$, which corresponds to $18.3 \times 10^{16}$ molecules $/ \mathrm{cm}^{2}$.

result, the peak intensity of the absorption bands in the amorphous film decreases considerably as compared with the spectrum of the isolated molecules (Fig. 1). Nevertheless, for the $\nu \mathrm{CO}$ vibrations, the peak intensity remains sufficiently high. This makes it possible to detect the absorption bands in the presence of background noise in Ur films of a considerably smaller thickness (10-20 nm).

Structuring of the silver surface can be accomplished by various methods. For example, for SERS, nanoclusters with a size of 50-100 nm have been formed on the surface of a single silver crystal photochemically using iodine vapor. ${ }^{26}$ In Ref. 15, silver nanoclusters of a size of 100-300 nm have been prepared by electrochemical deposition of a $40 \mathrm{~nm}$ silver layer on top of a $100 \mathrm{~nm}$ sputtered gold layer. In our experiment, after the electrochemical deposition of silver on the relatively smooth surface of the copper mirror (Fig. 2(a)), many nanostructures of a significantly larger size, 200-1000 nm (Fig. 2(b)), were present on the surface.

In the experimental spectrum of an Ur film with a thickness of about $11 \mathrm{~nm}$ (31 layers) deposited on the copper mirror, $\nu \mathrm{CO}$ and $\beta \mathrm{CH}$ vibration bands can be reliably identified (Fig. 3(a)). For a film with the same amount of Ur molecules on the nanostructured silver surface, we observed an enhancement of the integrated intensity of the $\nu \mathrm{CO}$ absorption bands by 3.5 fold and approximately fourfold enhancement for the $\beta \mathrm{CH}$ planar bending vibrations (Fig. 3(a)). Note that the absorption band corresponding to the $\beta \mathrm{NH}$ vibrations was not detected at all in the spectrum on the noise background (Fig. 3). In other experiments with the electrochemical nanostructuring of silver surface, ${ }^{15} 3-10$ fold intensity enhancement has been demonstrated for films of similar thickness. With increasing the Ur film thickness to $50 \mathrm{~nm}$ (150 layers) the intensity of the absorption bands corresponding to the $\nu \mathrm{CO}$ vibrations on the silver mirror was only 1.65 fold higher compared to that on the copper mirror (Fig. 3(b)). From the enhancement factors and the film thickness, the average enhancement factor $K_{x}$ for a fragment of a thick 


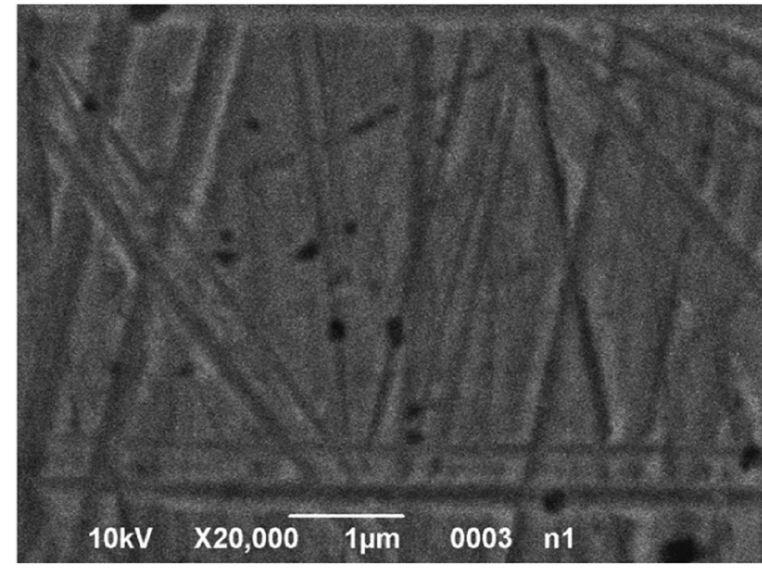

(a)

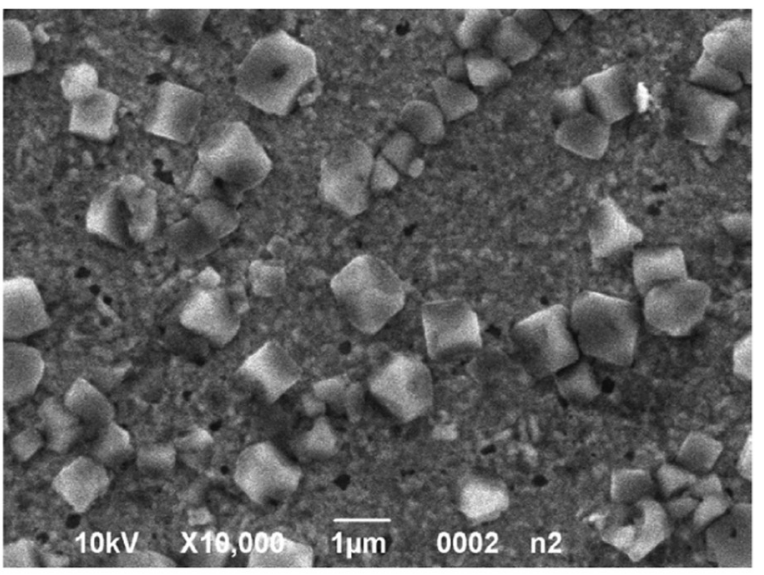

(b)

FIG. 2. Surface topography obtained using a JEOL scanning electron microscope: polished copper mirror (a); copper mirror after electrochemical silver plating (b). The scale is shown as a horizontal white bar.

film in the range of $12-60 \mathrm{~nm}$ can be determined using the equation

$$
K_{x}=K_{1}-\left(\frac{K_{0}-K_{1}}{n-1}\right),
$$

where $K_{0}$ and $K_{1}$ are the experimentally determined enhancement factors for thin and thick films and $n$ is the ratio of their thicknesses.

For $K_{0}=3.5$ and $K_{1}=1.65$, obtained by measuring the intensities of the $\nu \mathrm{CO}$ vibrational bands, and $n=5$, Eq. (1) yields $K_{x}=1.12$. The intensity of the absorption bands corresponding to the $\beta \mathrm{CH}$ and $\beta \mathrm{NH}$ bending vibrations remained small (even for the thick film) (Fig. 3(b)), thus they were not considered when evaluating the enhancement factors.

It should be noted that the nanostructured surface used in the experiment was not optimal for obtaining large enhancement of IR spectral bands. The largest enhancement in SEIRA is known to be obtained with 5-10 nm-thick metal island films deposited on a dielectric substrate. ${ }^{27}$ In such films the individual islands merge into relatively extended nanostructures on the verge of percolation. ${ }^{27}$ The shape of the absorption band becomes asymmetric, typical of the Fano resonance. ${ }^{27}$ For most large clusters studied by us,

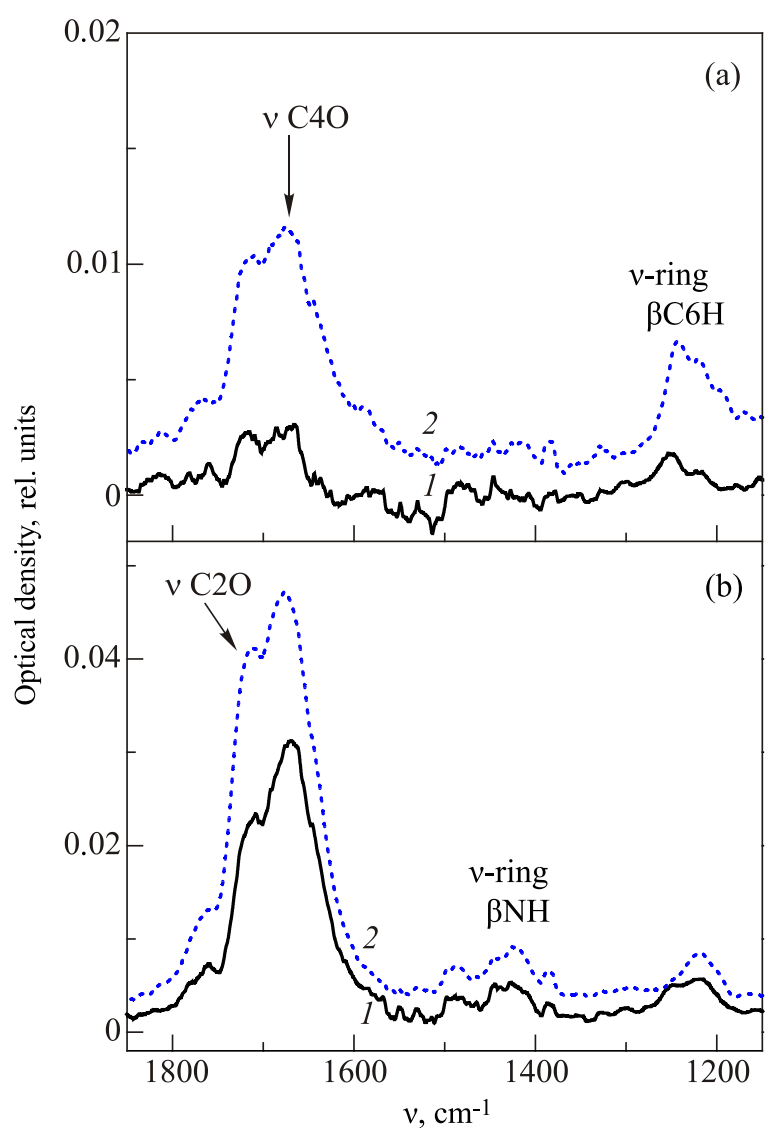

FIG. 3. FTIR absorption spectra of low-temperature $(T=6 \mathrm{~K})$ films of Ur molecules in the range of the $\nu \mathrm{CO}$ stretching vibrations and the vibrations of the ring: Ur film with a density of $1.7 \times 10^{-6} \mathrm{~g} / \mathrm{cm}^{2}$, which corresponds to 31 layers or a thickness of $11 \mathrm{~nm}$ (a); Ur film with a density of $8.5 \times 10^{-6}$ $\mathrm{g} / \mathrm{cm}^{2}$, which corresponds to 150 layers or a thickness of $50 \mathrm{~nm}$ (b). 1 -film on the copper mirror, 2-film on the surface of the nanostructured silver.

there was virtually no percolation (Fig. 2(b)). However, it can be assumed that some of the obtained clusters have large radii of local curvature. A rough estimate of the enhancement decay at a distance $d$ from a cluster of radius $R$ within the spherical model gives $(R /(R+d))^{12}$ for SERS ${ }^{2}$ and $(R /(R$ $+d))^{6}$ for SEIRA. ${ }^{28}$ Experiments also show that the enhancement in SERS decays much faster than that in SEIRA. ${ }^{29}$ Previously, it has been experimentally established that for a silver island film, the enhancement in SEIRA is reduced fivefold at a distance of $15 \mathrm{~nm} .{ }^{30}$ This result is in good agreement with our experimental results.

It is well-known that the interaction between a molecule and a metal nanoparticle already in the first adsorbed layer can cause significant changes in the intensity of individual oscillations. ${ }^{2}$ Therefore, to test the chemical mechanism of enhancement, we performed quantum-chemical calculations of the interaction energies between silver nanoclusters $\left(\mathrm{Ag}_{20}\right)$ and $\mathrm{Ur}$ monomers (U1_20Ag) and dimers (U2_20Ag) and calculated their vibrational spectra. The silver nanoclusters with this number of atoms are stable tetrahedral nanostructures with the $T_{d}$ symmetry. Using the method DFT/ B3LYP/BS1, we obtained the total energies, as well as interaction and relative energies for four complexes of U1_20Ag and five complexes of U2_20Ag (Table 1). The most energetically stable complexes U1_20Ag are characterized by the coordination bond between C4O (U1_20Ag_0), C2O (U1_20Ag_1) and a silver atom on top of the tetrahedral 
cluster (Fig. 4). In the most energetically stable complexes of dimers U2_20Ag (U2_20Ag_0 and U2_20Ag_1), only $\mathrm{C} 4 \mathrm{O}$ is bonded to top of the tetrahedron (Fig. 4). Calculations of the vibrational spectra showed that the formation of such complexes leads to an increase in the intensity of individual vibrations (Fig. 5). The intensity of the stretching vibration of the carbonyl $\mathrm{C} 4 \mathrm{O}$ group of the complex U1_20Ag_0 (Table 1), which is involved in the formation of the coordination bond (Fig. 5(a)), increased threefold. In experiment, the intensity of the $\nu \mathrm{C} 2 \mathrm{O}$ band increased only twice (Fig. 3(a)). The intensity of the $\beta \mathrm{N} 1 \mathrm{H}$ and $\beta \mathrm{C} 6 \mathrm{H}$ bending vibrations was also doubled (Fig. 5(a)). The U2_20Ag_0 complex showed a fivefold increase in the intensity of the $\nu \mathrm{C} 4 \mathrm{O}$ vibration (Fig. 5(b)). The formation of the coordination bond had practically no effect on the intensity of the $\beta \mathrm{NH}$ and $\beta \mathrm{CH}$ bending vibrations in an Ur dimer (Fig. 5(b)). Such manifestations of the chemical enhancement mechanism for the $\nu \mathrm{C} 2 \mathrm{O}$ bands should be considered in the study of the IR absorption of Ur monolayers. However, for the multilayer Ur films (with the number of layers greater than 30) considered in this study the effect of chemical enhancement did not exceed $10 \%$.

The average enhancement factor $K_{x}=1.12$ obtained for a fragment of the $50 \mathrm{~nm}$ film using Eq. (1) shows that for the nanostructured silver surface used in the experiment, the practical range of the electromagnetic enhancement does not exceed 10-30 $\mathrm{nm}$. These estimates are consistent with the experimental data for Ag surfaces prepared by both sputtering $^{6,30}$ and electrochemical deposition. ${ }^{15}$ Despite the rapid enhancement decay with increasing distance from the nanostructured surface, SEIRA can be used in the matrix isolation spectroscopy for studying small molecules with a planar structure. Molecules that can, similar to uracil, incorporate between the lattice planes of the inert matrix are characterized by narrow absorption bands in the IR spectra of the matrix. In the matrix absorption spectra of these molecules, the peak intensities of the spectral bands grow drastically, as compared with the absorption spectra of the films (Fig. 1). This further improves the signal-to-noise ratio in the experimental spectra and provides the prospect of studying thin matrices by SEIRA using even simple optical layouts with single reflection. However, for structurally non-rigid

TABLE 1. Total energy ( $E_{\text {tot }}$, a.u.), interaction energy between the silver nanoclusters $\left(\mathrm{Ag}_{20}\right)$ and uracil monomers or dimers $(I E, \mathrm{~kJ} / \mathrm{mol})$ and the relative stability of the complexes $\left(E_{\mathrm{rel}}, \mathrm{kJ} / \mathrm{mol}\right)$ calculated by the method DFT/B3LYP/BS1.

\begin{tabular}{lccc}
\hline \hline Complex & $E_{\text {tot }}$ & $I E$ & $E_{\text {rel }}$ \\
\hline Ag20-uracil monomer & & & \\
U1_20Ag_1 & -3355.931126 & -23.8 & 5.8 \\
U1_20Ag_0 & -3355.933343 & -29.6 & 0.0 \\
U1_20Ag_2 & -3355.927935 & -15.4 & 14.2 \\
U1_20Ag_3 & -3355.926011 & -10.3 & 19.3 \\
Ag20-uracil dimer & & & \\
U2_20Ag_2 & -3770.914997 & -34.7 & 12.6 \\
U2_20Ag_1 & -3770.917868 & -32.1 & 5.1 \\
U2_20Ag_3 & -3770.914018 & -34.0 & 15.2 \\
U2_20Ag_4 & -3770.912573 & -28.4 & 19.0 \\
U2_20Ag_0 & -3770.919814 & -32.5 & 0.0 \\
\hline \hline
\end{tabular}
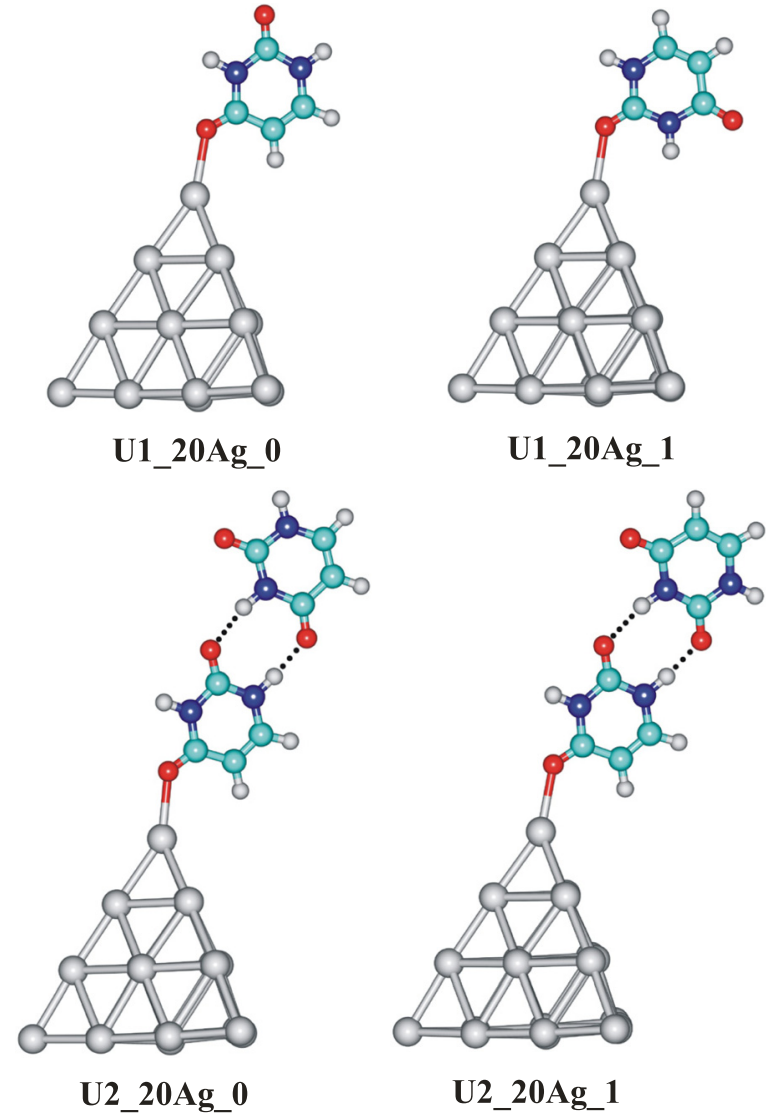

FIG. 4. Energetically most favorable structures of Ur monomers and dimers with 20-atom tetrahedral clusters of silver (see Table 1).

molecules, such as nucleosides, the absorption bands in the matrix spectra are much wider. ${ }^{18}$ Therefore, studying of these molecules by SEIRA in thin matrices requires optical layouts with multiple reflection ${ }^{15}$ or the use of nanoantenna arrays.

\section{Conclusions}

The study showed that a nanostructured silver surface obtained by a simple technique involving electrochemical deposition of silver on a copper mirror can provide 3-4 fold enhancement of the IR absorption of adsorbed molecules.

The enhancement effect by the nanostructured surface of the mirror decays with increasing the thickness of the adsorbed film, however, is observable even for films with a thickness of $10-30 \mathrm{~nm}$. The studies showed that it is possible to investigate the IR vibrational absorption spectra of molecules isolated in low-temperature inert-gas matrices of small thickness.

Quantum mechanical calculations showed that the coordination bond between the uracil and Ag nanoclusters leads to a 3-5 fold increase in the intensity of the $\nu \mathrm{CO}$ stretching vibrations and the vibrations of the ring in the first adsorbed layer of uracil molecules. However, in multilayer films, the main contribution to the increase in the vibrational intensity is caused by the electromagnetic mechanism.

The work was supported by the National Academy of Sciences of Ukraine (Grant No. 114U001070 and partially Grant No. 15/15H) and the National Foundation for Basic Research of Ukraine (Grant No. 54.1/044). The authors thank 


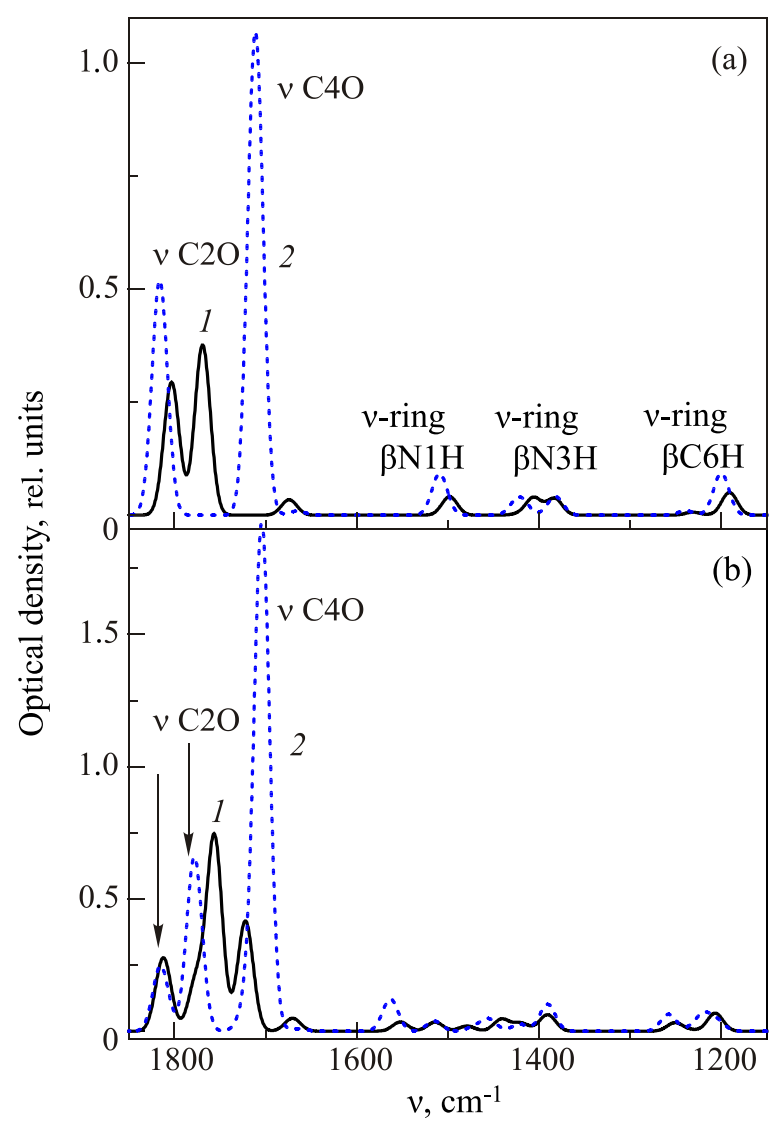

FIG. 5. Vibrational spectra of the complexes of Ur monomers and dimers with $\mathrm{Ag}$ clusters, calculated by DFT/B3LYP/BS1. The spectra of the Ur monomer $(1$, solid line) and U1_20Ag_0 complex (2, dashed line) (a). The spectra of the Ur dimer ( 1 , solid line) and the U2_20Ag_0 complex (2, dashed line) (b).

P. Mateychenko for the morphological measurements of the nanostructured silver surfaces and A. M. Plokhotnichenko and Yu. V. Rubin for a useful discussion.

a)Email: ivanov@ilt.kharkov.ua

${ }^{1}$ Surface Enhanced Raman Scattering, edited by R. K. Chang and T. E. Furtak (Plenum Press, New York, 1982).

${ }^{2}$ R. Aroca, Surface-Enhanced Vibrational Spectroscopy (Wiley, New York, 2006).

${ }^{3}$ Surface-Enhanced Raman Scattering: Physics and Applications, Topics in Applied Physics Vol. 103, edited by K. Kneipp, M. Moskovits, and H. Kneipp (Springer-Verlag, Berlin (2006).

${ }^{4}$ S. Schlucker, Angew. Chem. Int. Ed. 53, 4756 (2014).

${ }^{5}$ M. Fleischmann, P. J. Hendra, and A. J. McQuillan, Chem. Phys. Lett. 26, 163 (1974)

${ }^{6}$ D. L. Jeanmaire and R. P. Van Duyne, J. Electroanal. Chem. 84, 1 (1977).

${ }^{7}$ M. G. Albrecht and J. A. Creighton, J. Am. Chem. Soc. 99, 5215 (1977).
${ }^{8}$ A. Hartstein, J. R. Kirtley, and J. C. Tsang, Phys. Rev. Lett. 45, 201 (1980).

${ }^{9}$ M. Osawa and M. Ikeda, J. Phys. Chem. 95, 9914 (1991).

${ }^{10}$ T. R. Jensen, R. P. Van Duyne, S. A. Johnson, and V. A. Maroni, Appl. Spectrosc. 54, 371 (2000).

${ }^{11}$ F. Neubrech, A. Pucci, T. W. Cornelius, S. Karim, A. García-Etxarri, and J. Aizpurua, Phys. Rev. Lett. 101, 157403 (2008).

${ }^{12}$ A. Pucci, F. Neubrech, D. Weber, S. Hong, T. Toury, and M. L. de la Chapelle, Phys. Status Solidi B 247, 2071 (2010).

${ }^{13}$ R. Adato, A. A. Yanik, J. J. Amsden, D. L. Kaplan, F. G. Omenetto, Mi. K. Hong, S. Erramilli, and H. Altug, Proc. Natl. Acad. Sci. 106, 19227 (2009).

${ }^{14}$ R. Adato, A. A. Yanik, C.-H. Wu, G. Shvets, and H. Altug, Opt. Express 18, 4526 (2010).

${ }^{15}$ H. D. Wanzenböck, B. Mizaikoff, N. Weissenbacher, and R. Kellner, Fresenius' J. Anal. Chem. 362, 15 (1998).

${ }^{16}$ A. Les, L. Adamowicz, M. J. Nowak, and L. Lapinski, Spectrochim. Acta A 48, 1385 (1992).

${ }^{17}$ A. Yu. Ivanov, A. M. Plokhotnichenko, E. D. Radchenko, G. G. Sheina, and Yu. P. Blagoi, J. Mol. Struct. 372, 91 (1995).

${ }^{18}$ A. Yu. Ivanov, S. A. Krasnokutski, G. G. Sheina, and Yu. P. Blagoi, Spectrochim. Acta A 59, 1959 (2003).

${ }^{19}$ A. Yu. Ivanov and V. A. Karachevtsev, Fiz. Nizk. Temp. 33, 772 (2007) [Low Temp. Phys. 33, 590 (2007)].

${ }^{20} \mathrm{~A}$. Yu. Ivanov and A. M. Plokhotnichenko, Instrum. Exp. Tech. 52, 308 (2009).

${ }^{21}$ Electrochemistry Handbook, edited by A. M. Sukhotina (Khimia, Leningrad, 1981) [in Russian].

${ }^{22}$ Gaussian 09, Revision A.02, edited by M. J. Frisch, G. W. Trucks, H. B. Schlegel, G. E. Scuseria, M. A. Robb, J. R. Cheeseman, G. Scalmani, V. Barone, B. Mennucci, G. A. Petersson, H. Nakatsuji, M. Caricato, X. Li, H. P. Hratchian, A. F. Izmaylov, J. Bloino, G. Zheng, J. L. Sonnenberg, M. Hada, M. Ehara, K. Toyota, R. Fukuda, J. Hasegawa, M. Ishida, T. Nakajima, Y. Honda, O. Kitao, H. Nakai, T. Vreven, J. A. Montgomery, Jr., J. E. Peralta, F. Ogliaro, M. Bearpark, J. J. Heyd, E. Brothers, K. N. Kudin, V. N. Staroverov, R. Kobayashi, J. Normand, K. Raghavachari, A. Rendell, J. C. Burant, S. S. Iyengar, J. Tomasi, M. Cossi, N. Rega, J. M. Millam, M. Klene, J. E. Knox, J. B. Cross, V. Bakken, C. Adamo, J. Jaramillo, R. Gomperts, R. E. Stratmann, O. Yazyev, A. J. Austin, R. Cammi, C. Pomelli, J. W. Ochterski, R. L. Martin, K. Morokuma, V. G. Zakrzewski, G. A. Voth, P. Salvador, J. J. Dannenberg, S. Dapprich, A. D. Daniels, Ö. Farkas, J. B. Foresman, J. V. Ortiz, J. Cioslowski, and D. J. Fox (Gaussian, Inc., Wallingford, CT, 2009).

${ }^{23} \mathrm{~K}$. Irikura, Program SYNSPEC (National Institute of Standards and Technology, Gaithersburg, MD, USA, 1995).

${ }^{24}$ W. Saenger, Principles of Nucleic Acids Structure (Springer-Verlag, New York, 1984).

${ }^{25}$ O. Plekan, V. Feyer, A. Cassidy, V. Lyamayev, N. Tsud, S. Ptasinska, S. Reiff, R. G. Acres, and K. C. Prince, Phys. Chem. Chem. Phys. 17, 15181 (2015).

${ }^{26}$ Surface Enhanced Raman Scattering, edited by R. K. Chang and T. E. Furtak (Plenum Press, New York, 1982), p. 201.

${ }^{27}$ H. Nakashima, K. Kita, and Y. Suzuki, Adv. Appl. Phys. 2, 27 (2014).

${ }^{28}$ Near-Field Optics and Surface Plasmon Polaritons, Topics in Applied Physics Vol. 81, edited by M. Osawa and S. Kawata (Springer-Verlag, Berlin, Heidelberg, 2001), p. 163.

${ }^{29}$ R. Aroca and R. Bujalski, Vib. Spectrosc. 19, 11 (1999).

${ }^{30}$ E. Johnson and R. Aroca, J. Phys. Chem. 99, 9325 (1995).

Translated by L. Gardt 